\title{
МОБИЛЬНЫЕ СЕТИ 5G, КРИТЕРИИ ВЫБОРА СЕТИ И ИХ ПОСТРОЕНИЕ НА ОСНОВЕ МЕТОДА КОМПЛЕКСНОГО КРИТЕРИЯ
}

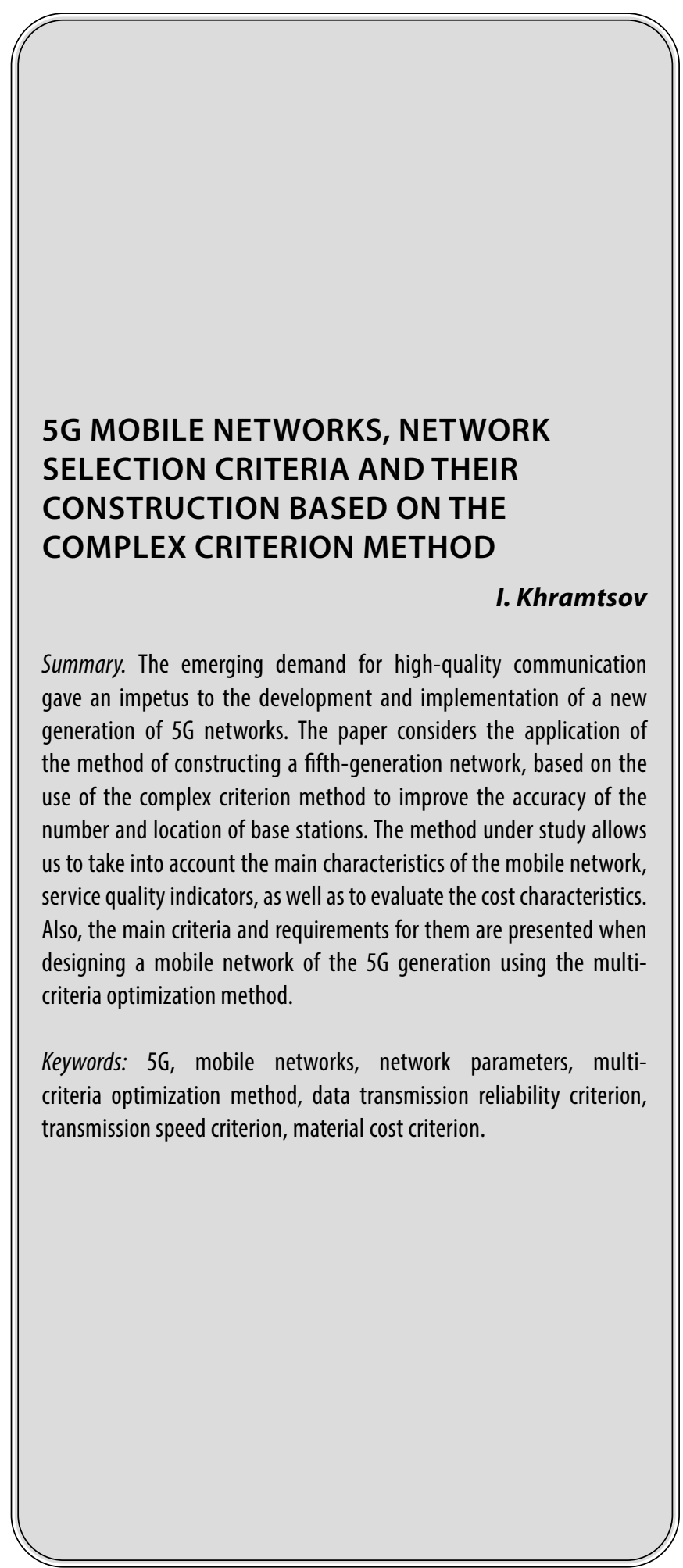

Храмцов Илья Александрович

Аспирант, Сибирский государственный университет науки и технологий имени академика М.Ф. Решетнева, г. Красноярск ilia.khramcov@yandex.ru

Аннотация. Возникший спрос на качественную связь дал толчок к развитию и внедрению сетей нового поклонения 5G. В материале работы рассмотрено применение метода построения сети пятого поколения, на основе использования метода комплексного критерия для повышения точности количества и местоположений базовых станций. Исследуемый метод позволяет учесть основные характеристики работы мобильной сети, показатели качества обслуживания, а также оценить стоимостные характеристики. Также, представлены основные критерии и требованиям к ним при проектировании мобильной сети поколения $5 \mathrm{G}$ с применением метода многокритериальной оптимизации.

Ключевые слова: 5G, мобильные сети, параметры сети, метод многокритериальной оптимизации, критерий надежности передачи данных, критерий скорости передачи, критерий материальных затрат. 


\section{Ввемение}

тремительное развитие технологий, рост конкуренции на рынке телекоммуникационных услуг, появление новых видов услуг в сетях мобильной связи, которые ранее были невозможны, сформировали новые требования потребителей к качеству предоставляемых им услуг. Данная ситуация дала старт к созданию сетей нового поколения (технология пятого поколения 5G. поэтому при создании сетей нового поколения важным моментом является соблюдение критериев оптимизации задач построения сети.

Основные проблемы и сложности при построении сети нового поколения можно объяснить отсутствием систематических универсальных критериев для решения задач оптимизации, которые нужно учитывать при создании сети.

Поэтому остро стоит вопрос в необходимости разработки актуальных методов определения структуры сети (оптимальной для решения поставленной задачи), которые будут учитывать основные критерии, обеспечивающие оценку стоимости качества обслуживания абонентского трафика.

\section{Основные критерии при создании сетей нового поколения}

Анализируя прошлые поколения сетей, при проектирование мобильных сетей 2G, одним из базовых критериев было качество голосовой связи и, согласно которому и проектировалась сеть [5].

Ключевым результатом при построении сети является выбор лучшего варианта оценки оптимальных показателей качества обслуживания с одной стороны и минимального количества сетевых ресурсов базовый сотовой станции с другой [4].

На стадии этапе проектирования, обычно есть данные по физическим параметрам среды - такие как распределенная застройка, плотная застройка, наличие или отсутствие деревьев, скорость движение абонента и другие. предполагаемое количество абонентов и параметры качества их обслуживания, например, скорость доступа к сети. Сложности в проектировании, на данном этапе, заключаются в том, что достаточно сложно оценить то количество ресурсов в мобильной сети, которые нужно выделить абоненту при оказании ему услуги сети. С другой стороны, для оператора связи необходимы минимальные затраты для обеспечения этих ресурсов. Поэтому, при проектировании сети учитывается стоимостная составляющая [1].
Поэтому важным стоит выделить, что при проектировании сетей мобильной связи важным является определение, отдельных критериев и их количества. Однако, стоит учесть, что анализ мобильной сети по нескольким критериям является достаточно и трудоемкой задачей, так как сеть по сути своей не однородна с точки зрения месторасположения и возможной загруженности сотовой базовых станций. Стоит отметить, что выбранные критерии могут быть зависимыми друг от друга и оказывать различное влияние на общие показатели работы сети. Для решения данной задачи при построения мобильной сети, необходимо исходить анализа возможных требований пользователей сети мобильной связи. Эти требования и будут являются ключевыми, на основе их будет формироваться оценка качества работы в проектируемой мобильной сети 5G. Качество связи можно будет оценить непосредственно по численному значению критерия.

Стоит сделать акцент на оценке несколько критериев показателей качества обслуживания пользователей, поэтому в данном случае целесообразно применить методы оценки многокритериальной оптимизации. Учитывая то, что при принятии решении будет учитываться сравнение вариантов по двум или более критериям с целью выделения эффективного варианта [11].

Рассмотрим применение метода оптимизации по Парето. Ключевым фактором будет свойство эффективности (в крайнем случае, слабого характера) где должно быть любое решение, претендующее на то, чтобы его назвали оптимальным.

Обобщим основные критерии проектирование мобильной сети поколения $5 \mathrm{G}$ с применением метода многокритериальной оптимизации [8]:

- выбор оптимальной сети поколения 5G, учитывая критерии, на основе которых она характеризуется;

- проектирование оптимальной структуры сети $5 \mathrm{G}$, то есть нахождения соответствующего расположения базовых станций;

- Выделения основных требований к выбору критериев для целей оптимизации на этапе планирования сети поколения $5 \mathrm{G}$.

Итак, критерии должны соответствовать следующим требованиям:

- их количество должно быть минимальным и включать в себя наиболее важные частные критерии;

- вектор оптимального решения для сети поколения $5 \mathrm{G}$ состоящий из множества числа частных критериев, должен с большей точностью описы- 
вать функциональную надежность проектируемой сети поколения $5 \mathrm{G}(\mathrm{K})$;

- частные критерии должны и характеризовать свойства сети поколения $5 \mathrm{G}$ и иметь простое содержание.

Важным фактором для сетей поколения 5G, отражающими параметры качества обслуживания потребителей, будет [2]:

- критерий надежности передачи данных (N). Так как он характеризуется вероятностью неработоспособности (Q) мобильной сети. При увеличении количества отказов отдельно взятой базовой станции надежность соединения снижается, что приводит к снижению надежности всей мобильной сети и качества, предоставляемой ею услуг;

- критерий скорости передачи (V) который является качественным показателем работоспособности сети связи 5G. Он характеризуется относительными отклонениями от нормы коэффициентов использования емкости кольца suring_N и сети $\Delta$ n network. Выбор данного параметра в качестве критерия основан на влиянии загрузки сети на ее надежность работы (так как если часть базовых станций работает с перегрузкой, а другая их часть простаивает либо недогружена, что приводит к дополнительным необоснованным затратам ресурсов в сети);

- критерий материальных затрат (С). Он характеризуется коэффициентом использования капитальных затрат Кикз на строительство базовых станций. Очевидно, что для операторов мобильной связи данный критерия должен быть минимальным.

Руководствуясь описанными выше критериями, сформируем задачу для разработки метода проектирования сети пятого поколения на основе комплексного критерия качества.

Каждый из выбранных вариантов построения сети мобильной связи Мі можно описать целевой функцией и функцией полезности [9].

$$
\begin{gathered}
\min K_{i}=\underline{\underline{K}}\left(N_{i}(\bar{x}), V_{i}\left(\bar{x}_{v}\right)\right), \\
C_{i}\left(\underline{\underline{\bar{x}_{x}}, \bar{x}_{\bar{x}}}\right) \leq C_{\text {dan }}
\end{gathered}
$$

где $K_{i}$ - вектор оптимального решения для сети мобильной связи $5 \mathrm{G} M_{i} ; N_{i}$ - частный критерий надежности передачи данных;

$V_{i}$ - частный критерий скорости передачи сети;
$C_{i}$ - частный критерий материальных затрат при создании сети; $\bar{x}_{N}$ - вектор надежности передачи данных;

$\bar{x}_{v}$ - вектор пропускной способности сети;

$C_{\text {доn }}$ - допустимое значение частого критерия материальных затрат.

В дальнейшем при планировании сети поколения $5 \mathrm{G}$ на этапе планирования и на этапе дальнейшей эксплуатации, данные векторы надежности передачи данных будет иметь вид [3,6]:

$$
\begin{aligned}
& \int 2 \leq \widetilde{\tau}_{\text {abubrous }} \leq 5 \mathrm{MKc} / \mathrm{\kappa M} \text {, } \\
& n_{\text {peCm_no:uni. }} \leq 2 \text {, } \\
& \left\{10^{-9} \leq B E R \leq 10^{-11}\right. \text {, } \\
& t_{\text {hebeETSNCP }} \leq 30 M C \text {, } \\
& t_{\text {nep pr.MSP.MS }-S P R i n g} \leq 50 \mathrm{MC} \text {. } \\
& \bar{x}_{N}=\left\{\begin{array}{l}
\rho_{a b} \\
\underline{\underline{P}}_{k} \\
\phi_{k}
\end{array}\right\}, \quad \bar{x}_{N}=\left\{\begin{array}{c}
\tau_{a b} \\
\chi^{m}
\end{array}\right\},
\end{aligned}
$$

Пропускную способность представим также в виде вектора:

$$
\bar{x}_{v}=\left\{\begin{array}{l}
\mu_{\text {link }} i j \\
\mu_{M U x_{-} i} \\
\mu_{\text {ring_N }} N \\
\mu_{\text {network }}
\end{array}\right\},
$$

где $\mu_{\text {link_ij, }} \mu_{M U X_{-} i}, \mu_{\text {ring_N }}, \mu_{\text {network }}-$ коэффициенты использования емкости канальных групповых трактов, мультиплексоров, колец и сети в целом [4].

Важно отметить ограничения при данной модели при использовании данных критериев - ограничением является использование исключительно кольцевых схем при построении сетей поколения $5 \mathrm{G}$.

Ограничения с учетом критерия надежности передачи данных, можно описать следующим образом

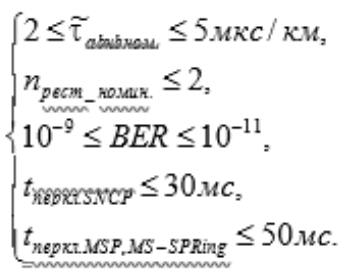

Для модели функциональной надежности, которые накладываются сектором телекоммуникаций и опера- 
торами сети нужно учитывать такие ограничения как [10]:

- среднее допустимое время прохождения сигнала сети любой пары узлов составляет 2-5 мкс/ кM;

- количество рестартов для одного соединения 2 (устанавливается оператором сети); - значение BER в диапазоне $10^{-9} \leq B E R \leq 10^{-11}$.

- полная вероятность работоспособности сети должна быть не меньше, чем 0.99999.

Для моделей с учетом критерия пропускной способности применяются следующие ограничения:

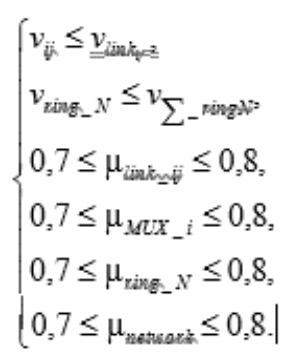

Для моделей материальных затрат при создании сети используют ограничения:

$$
\begin{aligned}
& \left\lceil C_{M O D} \leq C_{M A X_{-} M O D}\right. \\
& \left\{C_{N E} T W_{O R K} \leq C_{\text {MASRetsark: }}\right. \\
& \mid 15 \leq r \leq 30 \% \text {, } \\
& 3 \leq t \leq 5 \mathrm{rem} \text {. }
\end{aligned}
$$
сети;

Где $C_{M O D}$ - затраты оператора на модернизацию

$C_{\text {MAX_MOD }}$ - максимально возможные затраты оператора на модернизацию сети;

$C_{N E T W O R K}-$ капитальные затраты на проектирование сети;

$C_{\text {MAXNETWORK }}$ - максимально возможные инвестиционные вложения на строительство сети;

$r$ - величина среднерыночной прибыли среди операторов на рынке СНГ в период 2016-2018 гг; t - период окупаемости инвестиций.

Ключевым условием будет то, чтобы значения выбранных критериев должны быть положительными и больше нуля [6]:

$$
N_{i}>0, V>0, C_{i}>0 \text {; }
$$

- значения частных критериев и вектора стремятся к минимуму:

$$
N_{i}, V, C \rightarrow \min , K_{i} \rightarrow \min .
$$

При использовании метода многокритериальной оптимизации выбранные частные критерии позволяют в полной мере описать и оптимизировать параметры сети поколения $5 \mathrm{G}$.

Анализируя vl вариантов расположения базовых станций сети мобильной связи, приходим к выводу, что каждому вышеуказанному варианту соответствуют три состояния сети $S_{1}, S_{2}, S_{3}$ при которых частные критерии принимают значения $N_{i}, V_{i}, C_{i}$. Из предложенных вариантов руководствуясь обобщенными показателями качества обслуживания потребителей $g\left(v_{i}\right)$ необходимо выбрать оптимальный вариант. Именно основываясь на значении $g\left(v_{i}\right)$ будет выбран оптимальный вариант расположения базовых станций по трем частным критериям: надежности передачи данных, скорости передачи данных и величине материальных затрат.

Варианты расположения и соответствующие им значения критериев представим в виде матрицы G [8]:

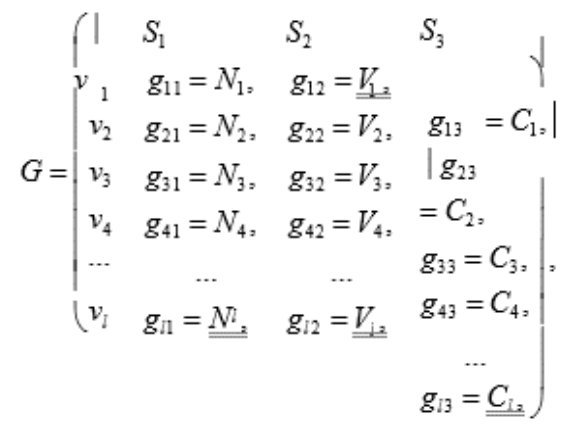

где $S_{1}, S_{2}, S_{3}$ - описывает состояния сети, при котором мобильная сеть принимает значения показателей надежности, скорости и материальных затрат;

$v_{1}, v_{2}, \ldots v_{i}$ - варианты расположения сотовых базовых станций, используемых для нахождения оптимального решения задачи.

Отметим, что используемые частые критерии могут иметь различную размерность, и для того, поэтому использовать метода оптимизации важным требованием будет выполнение нормирования значений матрицы G. Важно понимать, что масштабирования (нормирование) носит субъективный характер. В данном случае является приемлемы способ нормирования показателей на основе усреднения значения каждого показателя [4]:

$$
\sim \quad \frac{N_{\vartheta i}=}{\bar{N}} N_{i} \sim N, \frac{V_{i} i=\bar{V}}{\bar{V}}-\forall, \frac{C_{9 i}=C_{i}-C}{\bar{C}},
$$

Средние значения для каждого из частных критериев найдем исходя из количества вариантов расположения базовых станций: 


$$
\bar{N}=\frac{\sum_{i=1}^{L} W_{i}}{l}, \bar{V}=\frac{\sum_{i=1}^{j} i}{l}, \bar{C}=\frac{\sum_{i=1}^{i} C_{i}}{l},
$$

где $l$ - количество вариантов решений для выбранной сети мобильной связи $5 \mathrm{G}$.

Используя все варианты расположения базовых станций, выбранные с использованием как минимум трех вышеописанных методов, формируют область Парето в которой мобильная сеть будет планироваться с учетом целесообразных решений, при котором значе- ние каждого частного критерия не может быть улучшено без ухудшения других критериев.

\section{Зак^ючение}

Рассмотрев метод многокритериальной оптимизации как инструмент определения и проектирования структуры мобильной сети $5 \mathrm{G}$ можно сделать вывод что данный метод может использоваться для оптимизации расстановки базовых станций сети мобильной связи $5 \mathrm{G}$ при наличии большого количества альтернативных вариантов решений.

\section{ЛИТЕРАТУРА}

1. Беззатеев С.В., Федоров И.Р. Технология блокчейн в сетях 5G // Научно-технический вестник информационных технологий, механики и оптики. 2020. № 4. C. 472-484.

2. Данилов В.И. Сети и стандарты мобильной связи. СПбГУТ. 2015100 с.

3. Олейникова С.А., Токарев И.И. Сравнительный анализ моделей и методов решения задачи структурного синтеза для современных систем мобильной связи // Электроника и электротехника, № 4. 2018, С. 40-47.

4. Павловская К.А., Лозинская В.Н., Яремко И.Н. Многокритериальная оптимизация построения сетей пятого поколения на основе системного анализа // Вестник академии гражданской защиты. 2019. № 4(19). С. 111-117.

5. Полтева Т.В., Курилова А.А. «Дерево решений» как метод учёта неопределённости и риска при оценке эффективности инвестиционного проекта // Карельский научный журнал, Т. 7, № .4 (25), 2018, С. 106-109.

6. Стеклов В.К. Оптимизация и моделирование устройств и систем связи: учебник для высших учебных заведений / В.К. Стеклов, Л.Н. Беркман, Е.В. Кильчицкий.— Киев: Техника, 2004. - 576 с.

7. Чепцов М.Н., Климов В.В. Анализ оценок эффективности работы транспортной сети мобильного оператора связи // Сборник научных трудов ДонИЖТ. 2020. № 56. C. 43-48.

8. Чернышева Т.Ю., Жуков А.Г. Программный модуль учета рисков проекта на основе дерева решений // Ползуновский вестник. 2012. № 3-2. - С. 7073.

9. Agiwal M., Roy A., Saxena N. Next generation 5G wireless networks: A comprehensive survey // IEEE Communications Surveys \& Tutorials. 2016. V. 18. N3. P. 1617-1655. doi: 10.1109/COMST.2016.2532458

10. Isaev A.R. Future networks $5 G$ // Электронный мультидисциплинарный научный журнал с порталом международных научно-практических конференций Интернетнаука. 2016. № 9. С. 136-143.

11. Panwar N., Sharma S., Singh A.K. A survey on 5G: The next generation of mobile communication // Physical Communication. 2016. V. 18. P. 64-84. doi: 10.1016/j.phycom.2015.10.006 